\title{
INCIDENCE RELATIONS IN MULTICOHERENT SPACES. I
}

\section{BY}

\section{A. H. STONE}

1. Introduction. In a previous paper $\left({ }^{1}\right)$ a study was made of the relations between the numbers of components of unions and intersections of sets and their frontiers, in a space $S$ which was assumed to be connected, locally connected, and unicoherent. Here we shall investigate what happens when this last assumption is dropped. The results are naturally expressed in terms of $r(S)$, the "degree of multicoherence" of $S\left({ }^{2}\right)$. Roughly speaking, we shall find that most of the properties in [U] can indeed be carried over, except those on which the previous methods were based. Thus we shall require new methods, depending on devices of "decomposition" and "approximation" (cf. $\S \S 3,6$ ). A consequence of this is that, whereas no separation axioms were needed in the unicoherent case treated in [U], we shall now have to assume that $S$ is completely normal $\left({ }^{3}\right)$. On the other hand, this extra assumption will permit some relaxing of hypotheses elsewhere (for example, slightly weaker hypotheses on frontiers of sets). As in [U], we shall be able to consider subsets of $S$ which need not be open or closed, but are quite general.

In the first sections which follow (\$\$2-5) we consider the "natural" generalisations of several simple and useful point-set properties characteristic of unicoherence, notably the "Phragmén-Brouwer theorem" (cf. Kuratowski [2]). It is first shown that the expected equivalences break down in the multicoherent case, in general ( $\$ 2)$, though some implications survive. After developing the "decomposition" technique ( $\$ 3)$, we obtain a more complicated but valid generalisation of the Phragmén-Brouwer theorem ( $\$ 4$ ), and deduce (\$5) that the "natural" generalisations, considered in $\$ 2$, are after all valid if $S$ has no local cut points.

In the later sections, after developing the method of "approximation" ( $\$ 6)$, we investigate the properties of connected sets with disjoint frontiers, and related problems $(\$ \S 7,8)$. The contents of roughly the first half of [U] will

Presented to the Society, April 23, 1943, under the title Connectedness and coherence; received by the editors May $1,1948$.

(1) Stone [1]; this paper will be referred to as [U]. Numbers in brackets refer to the bibliography at the end of the paper.

(2) For the fundamental properties of $r(S)$, see Eilenberg [1], [2], [3] and Whyburn [1]. The definition of $r(S)$ is repeated below.

(3) That is, (i) $S$ is a $T_{1}$ space, (ii) every subspace of $S$ is normal. This could be weakened to assuming only that $S$ is normal (and $T_{1}$ ), without making any essential change, but at the cost of some slight complications; cf. 6.6(3) below. 
then have been generalised, and we shall leave the rest (which involves further complications) for a later paper.

Notations. Throughout, $S$ will denote a non-empty, connected, locally connected, completely normal $\left({ }^{3}\right)$ space, and capital letters will usually denote subsets of $S$. The "degree of multicoherence" of $S$ is defined by $\left({ }^{4}\right)$

(1) $r(S)=\sup \left\{b_{0}(A \cap B) \mid A, B\right.$ are closed, connected, and $\left.A \cup B=S\right\}$ where

(2) $b_{0}(X)=($ number of components of $X)-1$ if this number is finite, and $b_{0}(X)=\infty$ otherwise.

(Thus $r(S)=0$ if and only if $S$ is unicoherent.) It is not necessary to assume that $r(S)<\infty$, though little would be lost by doing so. It is known (Eilenberg [1]) that $r(S)$ is finite whenever $S$ is, for example, a polytope.

For other notations, see [U].

2. Some fundamental implications.

2.1. In [U, Theorem 1, 3.2], a number of properties of $S$ were listed, each of which was equivalent to the unicoherence of $S$. We begin by determining the implications between the "obvious" generalisations of them (corresponding properties being numbered in the same way as in [U]); as was remarked above, the generalised properties are by no means equivalent in general, though they will be so in an important special case (Theorem 6, 5.1). As in the unicoherent case, several of the implications could be greatly generalised (cf. Hall-Wallace [1, Theorem 1]).

2.2. THEOREM 1. Each of the following propositions implies the next, and (iii) and (ii) are equivalent, and (ii)' ${ }^{\prime}$ (iii)', (iv), (v) and (vi) are mutually equivalent; but there are no other implications between them, in general.

(i) $r(S) \leqq n\left({ }^{5}\right)$.

(iii) If $C$ and $D$ are disjoint connected sets such that $\operatorname{Fr}(C) \subset \operatorname{Fr}(D)$, then $b_{0}\{\operatorname{Fr}(C)\} \leqq n$.

(ii) If $E$ is simple $\left(^{6}\right), b_{0}\{\operatorname{Fr}(E)\} \leqq n$.

(ii)' If $E$ is open (or, equivalently, closed) and simple, $b_{0}\{\operatorname{Fr}(E)\} \leqq n$.

(iii)' If $C$ and $D$ are disjoint open connected sets such that $\operatorname{Fr}(C)=\operatorname{Fr}(D)$, then $b_{0}\{\operatorname{Fr}(C)\} \leqq n$.

(iv) If $G_{1}, G_{2}, \cdots, G_{n+2}$ are $n+2$ open connecled sets such that $\operatorname{Fr}\left(G_{i}\right)$ $\cap \operatorname{Fr}\left(G_{j}\right)=0$ whenever $i \neq j$, and if $p, q$ belong to the same component of $\cap\left\{G_{j} \mid j \neq i\right\}$ for each $i$, then $p$ and $q$ belong to the same component of $\cap G_{i}$ $(i, j=1,2, \cdots, n+2)$.

(v) If $F_{1}, F_{2}, \cdots, F_{n+2}$ are $n+2$ closed pairwise disjoint sets, the union of no $n+1$ of which separates two points $p, q$, then $U F_{i}$ does not separale $p$ and $q$ $(1 \leqq i \leqq n+2)$.

(4) Following Eilenberg [1]. It will be shown later (Theorem 10,7.7) that the sets $A, B$ in the definition of $r(S)$ could be taken to be open instead of closed.

(5) $n$ always denotes a non-negative (finite) integer.

(8) That is, both $E$ and $\operatorname{Co}(E)$ are connected ([U, 3.1]). 
(vi) If a (closed) set $A$ separates two points $p, q$, then so does the union of some $n+1$ (or fewer) components of $A$.

The proof that each proposition implies the next is a straightforward generalisation of the argument in [U, 3.2].

To prove that (ii) $\rightarrow$ (iii), let $C$ and $D$ be as in (iii), and let $E$ be the component of $\operatorname{Co}(C)$ which contains $D ; E$ is simple (cf. [U, 3.1(1)]), and it is easy to see that $\operatorname{Fr}(E)=\operatorname{Fr}(C)$, so that $b_{0}(\operatorname{Fr}(C)) \leqq n$ from (ii).

Incidentally, a similar argument shows that (iii)' is not affected if, instead of requiring $\operatorname{Fr}(C)=\operatorname{Fr}(D)$ in it, we require only $\operatorname{Fr}(C) \subset \operatorname{Fr}(D)$.

To prove that (vi) $\rightarrow$ (ii)', let $E$ be an open simple set, and suppose $b_{0}(\operatorname{Fr}(E))>n$; thus we can write $\operatorname{Fr}(E)=F_{1} \cup F_{2} \cup \ldots \cup F_{n+2}$, where the sets $F_{i}$ are closed, pairwise disjoint, and non-empty. Choose points $x_{i} \in F_{i}$, open sets $U_{i} \supset F_{i}$ such that $\bar{U}_{i} \cap \bar{U}_{j}=0$ whenever $i \neq j$, and connected neighbourhoods $V_{i}$ of the respective points $x_{i}$ such that $V_{i} \subset U_{i}$. There will now exist points $p \in E-U \bar{U}_{i}, q \in \operatorname{Co}(E)-U \bar{U}_{i}, y_{i} \in V_{j} \cap E$; and, since $E$ is open and connected, there will exist closed connected sets $K_{i} \subset E$ containing both $p$ and $y_{i}\left({ }^{7}\right)$. Let $W_{i}(1 \leqq i \leqq n+2)$ be an open set containing $F_{i}$ such that $\bar{W}_{i} \subset U_{i}-\cup K_{j}$. Then $\operatorname{Fr}\left\{\operatorname{Co}(E) \cup \cup W_{i}\right\}$ separates $p$ and $q$, and can be written as $U\left\{E \cap \operatorname{Fr}\left(W_{i}\right)\right\}$, a union of $n+2$ pairwise disjoint closed sets. $\mathrm{By}(\mathrm{vi})$, the union of some $n+1$ of them, say $U\left\{E \cap \operatorname{Fr}\left(W_{i}\right) \mid 2 \leqq i \leqq n+2\right\}$, must also separate $p$ and $q$. But it is disjoint from the connected set $K_{1} \cup V_{1}$ $\cup \mathrm{Co}(E)$, which contains both $p$ and $q$, giving a contradiction.

2.3. To see that (ii) does not imply (i), take $S$ to be a linear graph homeomorphic to a figure 8 ; it is easy to see that $r(S)=2$, while for every simple subset $E$ of $S$ we have $b_{0}(\operatorname{Fr}(E)) \leqq 1$.

Finally, to see that (ii)' does not imply (ii), we define $S$ as follows. Let $A$ be a 2 -cell (a circle plus its interior), and take six points $p_{1}, q_{1}, p_{2}, q_{2}, p_{3}, q_{3}$ in cyclic order on the boundary of $A$. Join $p_{i}$ to $q_{i}(i=1,2,3)$ by two simple $\operatorname{arcs} L_{i}, M_{i}$, in such a way that all six arcs are disjoint from each other and from $A$ except for their end points. Write $S=A \cup \cup\left(L_{i} \cup M_{i}\right)$. It is not hard to see that, if $E$ is an open simple subset of $S, \operatorname{Fr}(E)$ has at most 6 components. On the other hand, $A$ can be expressed as the union of two disjoint connected sets $X, Y$, in such a way that $p_{i} \in X$ and $q_{i} \in Y(i=1,2,3)\left({ }^{8}\right)$. Let $H_{i}, K_{i}$ be proper sub-arcs of $L_{i}, M_{i}$ respectively, containing $p_{i}$. Then, defining $E_{0}=X \cup \cup\left(H_{i} \cup K_{i}\right)$, we have that $E_{0}$ is simple; and $\operatorname{Fr}\left(E_{0}\right)$, consisting of $A$ plus a point on each arc, has 7 components. The proof of Theorem 1 is thus complete.

\section{Decompositions of sets and their frontiers.}

3.1. Before continuing with the study of the properties just considered, it is convenient to derive some theorems dealing (roughly speaking) with the

(7) This follows easily from, for example, Whyburn $[1$, p. 30$]$.

(8) For example, by taking $X$ and $Y$ to be complementary totally imperfect subsets of $E$; cf. Kuratowski [1, p. 267]. 
problem of extending a given decomposition of the frontier of a set, to one of the whole set. As these are of some independent interest, we shall derive them in rather stronger a form than will actually be needed below.

3.2. Lemma. Let $C$ be any subset of $S$; let $\left\{F_{1}, F_{2}, \cdots, F_{n}\right\}$ be any finite closed covering of $\operatorname{Fr}(C)\left({ }^{9}\right)$, and let $V_{j}$ be an open set containing $F_{j}(1 \leqq j<n)$. Then, if $N_{j}$ is the union of those components of $V_{j} \cap\left(\bar{C} \cup \cup F_{i}\right)$ which meet $F_{j}$, the set $\cup\left\{N_{j} \mid j<n\right\}-F_{n}$ is open relative to $\bar{C}$.

Let $x \in \bigcup N_{j}-F_{n}$ (where $j<n$ ) be given; we must construct a neighbourhood $U$ of $x$ such that $U \cap \bar{C} \subset \cup N_{j}-F_{n}$. If $x \in \operatorname{Int}(C)$, let $x \in N_{j}$ say, and take $U$ to be a connected neighbourhood of $x$ contained in $\left(V_{j} \cap C\right)-F_{n}$; clearly $U \subset N_{j}-F_{n}$. Thus we may assume $x \in \operatorname{Fr}(C)$. Let $x$ belong to exactly $k$ of the sets $F_{i}$; thus we may suppose $x \in F_{1} \cap F_{2} \cap \ldots \cap F_{k}-\left(F_{k+1} \cup \ldots\right.$ $\left.\cup F_{n}\right)$. Let $U$ be a connected neighbourhood of $x$ contained in $V_{1} \cap V_{2}$ $\cap \cdots \cap V_{k}$ and disjoint from $F_{k+1} \cup \ldots \cup F_{n}$. Then $U \cap \bar{C}=\{U \cap \operatorname{Fr}(C)\}$ $\cup\{U \cap \operatorname{Int}(C)\}$, and so

$$
U \cap \bar{C} \subset F_{1} \cup \ldots \cup F_{k} \cup \cup\left\{P_{\lambda} \mid P_{\lambda}\right. \text { is a component of }
$$

$$
\text { Int } \left.(C) \cap V_{1} \cap \cdots \cap V_{k} \text { which meets } U\right\} \text {. }
$$

Since $U$ also meets $\operatorname{Co}\left(P_{\lambda}\right)$ (in $x$ ), there exists for each $\lambda$ a point $y_{\lambda} \in U \cap \operatorname{Fr}\left(P_{\lambda}\right) \subset U \cap \operatorname{Fr}(C)$, since $U$ does not meet $\operatorname{Fr}\left(V_{i}\right)$ if $i \leqq k$. Thus $y_{\lambda} \in F_{j}$ for some $j \leqq k$. If $Q_{\lambda}$ is the component of $\left(\bar{C} \cup \cup F_{i}\right) \cap V_{j}$ which contains $y_{\lambda}$, clearly $P_{\lambda} \subset Q_{\lambda} \subset N_{j}$. Since also $F_{j} \subset N_{j}$, (1) shows that $U \cap \bar{C} \subset \cup N_{j}(j<n)$; and since also $U \subset \mathrm{Co}\left(F_{n}\right)$, the result follows.

Corollary. If $V$ is open and $V \cap F \mathrm{~F}(C)$ is closed, the union $N$ of those components of $V \cap \bar{C}$ which meet $\operatorname{Fr}(C)$ is open relative to $\bar{C}$.

3.3. Relative connectedness. For the convenience of the reader, we repeat the definition ([U,2.2]): A set $A$ is connected relative to $B$ if, whenever $X$ is a non-empty subset of $A$ which is both open and closed relative to $A, X$ and $B$ are not (mutually) separated. We shall make frequent use of the following obvious properties:

(1) If $A$ is connected relative to $B$, and $A \supset B$, and if $B$ is connected, then so is $A$.

(2) If $A \supset B \supset C$, and if $A$ is connected relative to $B$, and $B$ is connected relative to $C$, then $A$ is connected relative to $C$.

3.4. Theorem $2\left({ }^{10}\right)$. Let $C$ be any subset of $S$, and let $\left\{F_{1}, F_{2}, \cdots, F_{n}\right\}$ be a finite closed covering of $\operatorname{Fr}(C)$ of order at most $m\left({ }^{11}\right)$, such that $U F_{i} \neq 0$.

(') That is, each $F_{i}$ is closed and $U F_{i} \supset \operatorname{Fr}(C)$.

(10) In the case $C=S, m=1, n=2, F_{i}$ connected, this theorem reduces to the characterisation of local connectedness given in Wilson [1].

(i1) That is, no $m+1$ of the sets $F_{i}$ have a common point. 
Let open sets $U_{i} \supset F_{i}$ be given. Then there exist closed sets $H_{i}(1 \leqq i \leqq n)$ forming a covering of $C$ of order at most $m+1$, such that

(a) $H_{i} \supset F_{i}$, and $H_{i}$ is connected relative to $F_{i}$,

(b) $U H_{i}=\bar{C} \cup \cup F_{i}$,

(c) $H_{i} \cap U F_{k} \subset U_{i}$,

(d) $H_{i_{1}} \cap H_{i_{2}} \cap \cdots \cap H_{i_{m+1}} \subset \operatorname{Int}(C)-U F_{k}$,

(e) $\operatorname{Fr}\left(H_{i_{1}}\right) \cap \operatorname{Fr}\left(H_{i_{2}}\right) \cap \cdots \cap \operatorname{Fr}\left(H_{i_{m+1}}\right)=0$.

$\left(1 \leqq i_{1}<i_{2}<\cdots<i_{m+1} \leqq n ; 1 \leqq i, k \leqq n\right.$.)

The proof goes by induction over $n, m$ remaining fixed throughout. When $n=1$, the assertion is trivial (take $H_{1}=\bar{C} \cup F_{1}$; note that in any case $m \geqq 1$ ). Now assume the theorem true for $n-1$, where $n \geqq 2$.

There is evidently no loss in assuming that $F_{1} \neq 0$ and that

(1) $\bar{U}_{i_{1}} \cap \bar{U}_{i_{2}} \cap \cdots \cap \bar{U}_{i_{m+1}}=0$ whenever $1 \leqq i_{1}<i_{2}<\cdots<i_{m+1} \leqq n$.

Choose open sets $V_{i}$ such that $F_{i} \subset V_{i} \subset \bar{V}_{i} \subset U_{i}$, and let $N_{i}$ be the union of those components of $V_{i} \cap\left(\bar{C} \cup U F_{k}\right)(1 \leqq k \leqq n)$ which meet $F_{i}$; thus $U_{i} \supset \bar{N}_{i} \supset N_{i} \supset F_{i}$, and $N_{i}$ is connected relative to $F_{i}$.

Consider the components $Y_{\lambda}$ (say) of $C-\left\{\cup N_{j} \cup F_{n}\right\}$. (We shall reserve the symbol $j$ for a suffix running from 1 to $n-1$.) Let $L$ be the union of those sets $Y_{\lambda}$ for which $\operatorname{Fr}\left(Y_{\lambda}\right)$ meets $F_{n}$, and let $M$ be the union of the others; thus $L \cup M=C-\left\{\cup N_{j} \cup F_{n}\right\}$ and $L \cap M=0$. Further, since in any case $\operatorname{Fr}\left(Y_{\lambda}\right) \subset \operatorname{Fr}(C) \cup \cup \operatorname{Fr}\left(N_{j}\right) \cup F_{n}$, we have $\operatorname{Fr}(M) \subset \cup \bar{N}_{j}$. The sets $\bar{N}_{j}$ form a covering of $\operatorname{Fr}(M)$ of order at most $m$ (from (1)); hence we can apply the induction hypothesis to obtain closed sets $H_{j}(1 \leqq j<n)$, no $m+2$ of which have a common point, such that

(2) $H_{j} \supset \bar{N}_{j} ; H_{j}$ is connected relative to $\bar{N}_{j} ; \cup H_{j}=\bar{M} \cup \cup \bar{N}_{j} ; H_{j}$ $\cap\left(\bar{N}_{1} \cup \ldots \cup \bar{N}_{n-1}\right) \subset U_{j} ;$ and $H_{j_{1}} \cap H_{j_{2}} \cap \cdots \cap H_{j_{m+1}} \subset \operatorname{Int}(M)$ whenever $j_{1}, \cdots, j_{m+1}$ are all distinct (and less than $n$ ).

Define $H_{n}=\bar{L} \cup F_{n}$. We shall first show that $H_{1}, H_{2}, \cdots, H_{n}$ have the desired properties except for (e), and shall then show that property (e) can also be obtained by modifying the sets $H_{i}$.

To show that no $m+2$ of the sets $H_{i}$ can have a common point, it will suffice to verify that $H_{n} \cap H_{1} \cap H_{2} \cap \cdots \cap H_{m+1}=0$. But $H_{1} \cap H_{2} \cap \ldots$ $\cap H_{m+1} \subset \operatorname{Int}(M)$, from (2); and $H_{n} \cap \operatorname{Int}(M)=\left(\bar{L} \cup F_{n}\right) \cap \operatorname{Int}(M)=0$ since $L \cap M=0=F_{n} \cap M$.

Properties (a) and (b) are immediate, in view of 3.3(2). Thus, since (d) is an immediate consequence of (c) and (1), we have only to verify (c). Suppose first that $i<n$. Then $H_{i} \cap F_{n} \subset\left(\bar{M} \cap F_{n}\right) \cup \cup \bar{N}_{j} \subset \operatorname{Fr}(M) \cup \cup \bar{N}_{j}=\cup \bar{N}_{j}$; thus, from (2), $H_{i} \cap U F_{k} \subset H_{i} \cap U \bar{N}_{j} \subset U_{i}$. And, if $i=n$, we have $H_{n} \cap U F_{k} \subset F_{n}$ $\cup\left\{\bar{L} \cap\left(U N_{j}-F_{n}\right)\right\}$; but since $U N_{j}-F_{n}$ is open relative to $\bar{C}$, from the Lemma (3.2), and is disjoint from $L$, it is also disjoint from $\bar{L}$, giving $H_{n} \cap \bigcup F_{k}$ $\subset F_{n} \subset U_{n}$.

Finally, we must modify the sets $H_{i}$ so that they satisfy (e). There exist 
open sets $X_{i} \supset H_{i}$ such that (i) no $m+2$ of the sets $\bar{X}_{i}$ have a common point, (ii) $\bar{X}_{i} \subset U_{i} \cup \operatorname{Co}\left(\cup F_{k}\right)$. Let $K_{i}$ be the union of those components of $X_{i}$ $\cap\left(\bar{C} \cup \cup F_{k}\right)$ which meet $H_{i}$, and write $H_{i}^{*}=\bar{K}_{i}$. The sets $H_{i}^{*}$ have all the properties of the sets $H_{i}$ (that is, no $m+2$ of them have a common point, and (a)-(d) hold for them). Further, they satisfy (e). For suppose there exists a point $x \in \operatorname{Fr}\left(H_{1}^{*}\right) \cap \operatorname{Fr}\left(H_{2}^{*}\right) \cap \ldots \cap \operatorname{Fr}\left(H_{m+1}^{*}\right)$ (say); then $x \in \bar{X}_{1} \cap \ldots$ $\cap \bar{X}_{m+1}$, so from (i) $x \in H_{m+2} \cup \cdots \cup H_{n}$, and from (ii) $x \in \operatorname{Int}(C)$. Thus $x$ belongs to some $H_{i}$ for $i \leqq m+1$, say to $H_{1}$, and consequently belongs to some component $P$ of $X_{1} \cap \operatorname{Int}(C)$. Clearly $P$ is open and is contained in $K_{1}$; hence $x \in \operatorname{Int}\left(H_{1}^{*}\right)$-a contradiction.

3.5. Theorem 3. Let $C$ be any proper non-empty subset of $S$, and let $X$ be any closed set containing $\operatorname{Fr}(C)$ and connecled relative to $\operatorname{Fr}(C)$ (for example, $X=\operatorname{Fr}(C))$. Suppose $b_{0}(X) \geqq n \geqq 0$. Then there exist two closed sets $H, K$, each connected relative to $\operatorname{Fr}(C)$, such that $H \cup K=\bar{C} \cup X, \operatorname{Fr}(H) \cap \operatorname{Fr}(K)=0$, $H \cap K \subset \operatorname{Int}(C)-X$, and $b_{0}(H \cap K)+b_{0}(\bar{C} \cup X) \geqq n-1$.

Proof. We may write $X=F_{1} \cup F_{2} \cup \ldots \cup F_{n+1}$, where the sets $F_{i}$ are closed, non-empty, and pairwise disjoint. By Theorem 2, there exist closed sets $H_{i}(1 \leqq i \leqq n+1)$ such that

(1) $H_{i} \supset F_{i} ; H_{i}$ is connected relative to $F_{i} ; \cup H_{i}=\bar{C} \cup X ; \operatorname{Fr}\left(H_{i}\right) \cap \operatorname{Fr}\left(H_{j}\right)$ $=0$ and $H_{i} \cap H_{j} \subset$ Int $(C)-X$ if $i \neq j$; and $H_{i} \cap H_{j} \cap H_{k}=0$ if $i, j, k$ are all distinct.

Form a linear graph $G$ having $n+1$ vertices $h_{i}(1 \leqq i \leqq n+1), h_{i}$ and $h_{j}$ being joined (by a 1-cell of $G$ ) if and only if $H_{i} \cap H_{j} \neq 0$. (That is, $G$ is the nerve of the system $\left\{H_{i}\right\}$.) Let $G$ have $\nu$ components $G_{\lambda}(1 \leqq \lambda \leqq \nu)$; we may suppose that $G_{\lambda}$ has vertices $h_{i_{\lambda-1}+1}, h_{i_{\lambda-1+2}}, \cdots, h_{i_{\lambda}}$ (where $0=i_{0}$ $\left.<i_{1}<\cdots<i_{\nu}=n+1\right)$. Let $E_{\lambda}=\bigcup\left\{H_{i} \mid i_{\lambda-1}<i \leqq i_{\lambda}\right\}$; thus the sets $E_{1}, \cdots, E_{\nu}$ are closed, non-empty, and pairwise disjoint, and their union is $\bar{C} \cup X$, so that

(2) $b_{0}(\bar{C} \cup X) \geqq \nu-1$.

Now let $T_{\lambda}$ be a connected subgraph of $G_{\lambda}$ having the same vertices as $G_{\lambda}$ and as few 1-cells as possible; thus $T_{\lambda}$ is a tree having $i_{\lambda}-i_{\lambda-1}$ vertices, and consequently having $\left(i_{\lambda}-i_{\lambda-1}-1\right) 1$-cells. There is a unique path joining each vertex $h_{i}$ of $G_{\lambda}$ (other than $h_{i_{\lambda-1+1}}$ ) to $h_{i_{\lambda-1+1}}$ in $T_{\lambda}$; let $K_{\lambda}$ be the set of all vertices of $G_{\lambda}$ for which this path has an odd number of 1-cells, and let $\mathfrak{F C}_{\lambda}$ consist of the remainder. We may suppose the notation so chosen that $h_{i} \in \mathcal{H}_{\lambda}$ for $i_{\lambda-1}<i \leqq j_{\lambda}$, and $h_{i} \in K_{\lambda}$ for $j_{\lambda}<i \leqq i_{\lambda}$ (where $i_{\lambda-1}<j_{\lambda} \leqq i_{\lambda}$; naturally $K_{\lambda}$ may be empty). Define $H=\bigcup\left\{H_{p} \mid i_{\lambda-1}<p \leqq j_{\lambda}, 1 \leqq \lambda \leqq \nu\right\}, K=\bigcup\left\{H_{q} \mid j_{\lambda}\right.$ $\left.<q \leqq i_{\lambda}, 1 \leqq \lambda \leqq \nu\right\}$. From (1), $H$ and $K$ are closed sets, connected relative to $\operatorname{Fr}(C)$, and $\operatorname{Fr}(H) \cap \operatorname{Fr}(K)=0, H \cup K=\bar{C} \cup X, H \cap K \subset \operatorname{Int}(C)-X$. Further, $H \cap K=\bigcup\left\{H_{p} \cap H_{q}\right\}$, a union of closed sets which are pairwise disjoint (for different pairs $(p, q)$ ), from (1). And the number of these sets $H_{p} \cap H_{q}$ which are non-empty is the total number of 1-cells of all the $G_{\lambda}$ 's which have one 
end point in $\mathfrak{F C}_{\lambda}$ and the other in $K_{\lambda}$ Since every 1-cell in $T_{\lambda}$ is included here, by construction, we obtain

$$
b_{0}(H \cap K)+1 \geqq \sum\left(i_{\lambda}-i_{\lambda-1}-1\right)=n+1-\nu ;
$$

and the result now follows from (2).

3.6. THEOREM 4. Let $C$ be any subset of $S$, having components $\left\{D_{\mu}\right\}$, and $X$ be any closed set containing $\operatorname{Fr}(C)$ and connected relative to $\mathrm{Cl}(\mathrm{Co}(C))\left({ }^{12}\right)$. Then

$$
b_{0}(X) \leqq \sum b_{0}\left\{\operatorname{Fr}\left(D_{\lambda}\right)\right\}+b_{0}(\bar{C} \cup X)\left({ }^{13}\right) .
$$

Let $X=Y_{1} \cup Y_{2} \cup \cdots \cup Y_{k+1}$, where the sets $Y_{i}$ are closed, non-empty, and pairwise disjoint; it will evidently suffice to prove that

$$
k \leqq \sum b_{0}\left\{\operatorname{Fr}\left(D_{\lambda}\right)\right\}+b_{0}(\bar{C} \cup X),
$$

and in doing this we can certainly assume the right-hand side to be finite, so that $C$ can have only a finite number of components, say $D_{1}, D_{2}, \cdots, D_{n}$ whose frontiers are not connected. Let $\left\{E_{\mu}\right\}$ be the remaining components of $C$ (with connected frontiers). The proof will go by induction over $n$.

Suppose first $n=0$. Write $H_{i}=Y_{i} \cup \cup\left\{E_{\mu} \mid \operatorname{Fr}\left(E_{\mu}\right) \subset Y_{i}\right\} \quad(1 \leqq i \leqq k+1)$. Clearly $\cup H_{i}=X \cup C=X \cup \bar{C}$, and each $H_{i}$ is closed and non-empty. Further, the sets $H_{i}$ are pairwise disjoint, since if (say) $H_{1} \cap H_{2} \neq 0$, there is no loss of generality in assuming that $Y_{2}$ meets some $E_{\mu}$ with $\operatorname{Fr}\left(E_{\mu}\right) \subset Y_{1}$, and then $Y_{2} \cap \operatorname{Int}\left(E_{\mu}\right)$ is a non-empty and relatively open-closed subset of $X$ which is separated from $\mathrm{Cl}(\mathrm{Co}(C))$, contrary to hypothesis. Hence $b_{0}(\bar{C} \cup X) \geqq k$, establishing (1) in this case.

Now suppose that (1) holds for $n-1$ (for all sets $C$ and $X$ satisfying the conditions), and write $C^{\prime}=C-D_{n}$. Then $X$ is a fortiori connected relative to $\mathrm{Cl}\left(\mathrm{Co}\left(C^{\prime}\right)\right)$, and contains $\operatorname{Fr}\left(C^{\prime}\right)$, so that the hypothesis of induction gives the existence of a (finite) non-negative integer $r \leqq b_{0}\left(\bar{C}^{\prime} \cup X\right)$ such that

$$
k \leqq r+\sum b_{0}\left\{\operatorname{Fr}\left(D_{i}\right)\right\} \quad(1 \leqq i \leqq n-1) .
$$

We can write $C^{\prime} \cup X=\bar{C}^{\prime} \cup X=K_{1} \cup K_{2} \cup \ldots \cup K_{r+1}$, a union of $r+1$ closed, non-empty, pairwise disjoint sets. Let $\operatorname{Fr}\left(D_{n}\right)$ meet exactly $s$ of the sets $K_{j}$, say $K_{1}, \cdots, K_{s}$ (where $\left.0 \leqq s \leqq r+1\right)$. Then, since $\operatorname{Fr}\left(D_{n}\right) \subset X \subset \cup K_{j}$, we have $b_{0}\left\{\operatorname{Fr}\left(D_{n}\right)\right\} \geqq s-1$.

Again, $\bar{D}_{n}$ is disjoint from $K_{j}$ if $j>s$; for otherwise $K_{j} \cap$ Int $\left(D_{n}\right)$ is a nonempty and relatively open-closed subset of $X$ which is separated from $\mathrm{Cl}(\mathrm{Co}(C))$, contrary to hypothesis. Accordingly, we have $\bar{C} \cup X$ $=\left(\bar{D}_{n} \cup K_{1} \cup K_{2} \cup \ldots \cup K_{s}\right) \cup K_{s+1} \cup \ldots \cup K_{r+1}$, a union of $r-s+2$ pairwise disjoint, closed, non-empty sets, showing that $b_{0}(\bar{C} \cup X) \geqq r-s+1$.

(12) To simplify the printing of complicated expressions, we shall sometimes write $\mathrm{Cl}(\dot{x})$ instead of $\bar{x}$.

${ }^{(13)}$ If $S$ is unicoherent, it can be shown that this inequality becomes an equality. 
Thus $b_{0}(\bar{C} \cup X)+b_{0}\left\{\operatorname{Fr}\left(D_{n}\right)\right\} \geqq r$, whence (1) follows from (2) in the present case also. This establishes (1) for all $n$, proving the theorem.

4. Extension of the Phragmén-Brouwer Theorem.

4.1. Let $A$ be an arbitrary connected set, and let $C$ be any component of $\operatorname{Co}(A)$ (so that $C$ is simple). We have seen (Theorem $1,2.2)$ that $\{r(S) \leqq n\}$ $\rightarrow\left\{b_{0}(\operatorname{Fr}(C)) \leqq n\right.$, for all $A$ and $\left.C\right\}$, though the converse implication is false in general $\left({ }^{14}\right)$. But a stronger result, which does characterize $r(S)$, can be obtained by considering all the components of $\operatorname{Co}(A)$ :

Theorem 5. If $A$ is any subset of $S$ such that $\bar{A}$ is connected and if $\left\{C_{\lambda}\right\}$ are the components of $\mathrm{Co}(A)$, then

$$
\sum b_{0}\left\{\operatorname{Fr}\left(C_{\lambda}\right)\right\} \leqq r(S) .
$$

Conversely, if for some fixed $n$ we have

$$
\sum b_{0}\left\{\operatorname{Fr}\left(C_{\lambda}\right)\right\} \leqq n
$$

whenever $\left\{C_{\lambda}\right\}$ are the components of the complement of an open connected set $A$ which is such that $b_{0}(\operatorname{Co}(A)\}<\infty$, then $r(S) \leqq n$. In this statement, the word "open" may be replaced by "closed."

4.2. In proving (1), we can assume $\operatorname{Fr}\left(C_{\lambda}\right) \neq 0$-for otherwise $C_{\lambda}=S$ and (1) holds trivially - and can thus take for each $\lambda$ an integer $n_{\lambda}$ such that $0 \leqq n_{\lambda} \leqq b_{0}\left\{\operatorname{Fr}\left(C_{\lambda}\right)\right\}$. It will clearly suffice to prove

$$
\sum n_{\lambda} \leqq r(S) \text {. }
$$

From Theorem 3 (3.5) there exist closed sets $H_{\lambda}, K_{\lambda}$, connected relative to $\operatorname{Fr}\left(C_{\lambda}\right)$, such that $H_{\lambda} \cup K_{\lambda}=\bar{C}_{\lambda}, H_{\lambda} \cap K_{\lambda} \subset$ Int $\left(C_{\lambda}\right)$, and $b_{0}\left(H_{\lambda} \cap K_{\lambda}\right) \geqq n_{\lambda}-1$. Write $L=\bar{A} \cup \cup H_{\lambda}, M=\bar{A} \cup \cup K_{\lambda}$. Then $L$ is connected relative to $\bar{A}$, and is therefore connected; similarly, so is $M$. Again, $L$ is closed. For suppose $x \in \bar{L}-L$; then $x \in \bar{A}$, so $x \in \operatorname{Int}\left(C_{\lambda}\right)$ for some $\lambda$, whence $x$ belongs to the closure of $\operatorname{Int}\left(C_{\lambda}\right) \cap L \subset H_{\lambda}$, and so $x \in H_{\lambda} \subset L$, a contradiction. Similarly $M$ is closed. Since $L \cup M=S$, we have $b_{0}(L \cap M) \leqq r(S)$.

Now $L \cap M=\bar{A} \cup \cup\left(H_{\lambda} \cap K_{\mu}\right)=\bar{A} \cup \cup\left(H_{\lambda} \cap K_{\lambda}\right)$, since if $\lambda \neq \mu$ we have $H_{\lambda} \cap K_{\mu} \subset \bar{C}_{\lambda} \cap \bar{C}_{\mu} \subset \bar{A}$. Further, $H_{\lambda} \cap K_{\lambda}=\operatorname{Int}\left(C_{\lambda}\right) \cap(L \cap M)$, so that $H_{\lambda} \cap K_{\lambda}$ is both open and closed relative to $L \cap M$. Thus $L \cap M$ has at least $1+\sum n_{\lambda}$ components (consisting of $\bar{A}$ together with the separate components of the sets $\left.H_{\lambda} \cap K_{\lambda}\right)$; that is, $r(S) \geqq b_{0}(L \cap M) \geqq \sum n_{\lambda}$, Q.E.D.

CoRollary. If $\bar{A}$ is connected and $r(S)<\infty$, all but at most a finite number of the components of $\operatorname{Co}(A)$ have connected frontiers.

4.3. We shall derive the remainder of Theorem 5 from the following sharper result:

(14) It holds if $n=0$, the resulting equivalence constituting the Phragmén-Brouwer theorem; cf. Kuratowski [2] and [U, Theorem 1, 3.2]. 
THEOREM 5a. If $r(S)>n$, there exists a connected set $A$ such that $b_{0}\{\operatorname{Co}(A)\}$ $<\infty$ and $b_{0}\{\operatorname{Fr}(A)\}>b_{0}\{\operatorname{Co}(A)\}+n$; further, $A$ can be taken to be open, or to be closed.

In fact, once Theorem $5 \mathrm{a}$ is established, Theorem 4 (3.6) shows that (2) above will be violated, and the proof of Theorem 5 will be complete.

First we construct an open set $A$ fulfilling the requirements of Theorem 5a. Since $r(S)>n$, there exist closed connected sets $A_{1}, B_{1}$ such that $A_{1} \cup B_{1}$ $=S$ and $A_{1} \cap B_{1}=D_{1} \cup D_{2} \cup \ldots \cup D_{n+2}$, where the sets $D_{i}$ are closed, nonempty, and pairwise disjoint. There exist open sets $U_{i} \supset D_{i}$ such that $\bar{U}_{i} \cap \bar{U}_{j}$ $=0$ if $i \neq j$; and we can further suppose that every component of $U_{i}$ meets $D_{i}$ (by discarding the other components of $U_{i}$ ). Write $A^{\prime}=A_{1} \cup \cup U_{i}, B^{\prime}=B_{1}$ $\cup \cup U_{i}$, and $X=\cup \bar{U}_{i}$. Clearly $A^{\prime}$ and $B^{\prime}$ are connected open sets, and $\operatorname{Fr}\left(A^{\prime}\right) \subset X$. Further, if $V$ is any component of any $U_{i}$, then since $V \cap B^{\prime}$ and $B^{\prime}-V$ are non-empty, they are not separated, from which it readily follows that $\operatorname{Fr}(V)$ meets $\operatorname{Fr}\left(A^{\prime}\right)$. Hence it is easy to see that $X$ is connected relative to $\operatorname{Fr}\left(A^{\prime}\right)$. Thus we may apply Theorem 4 (3.6) to the set $\mathrm{Co}\left(A^{\prime}\right)$ with components $\left\{C_{\lambda}\right\}$ (say), obtaining

$$
b_{0}(X) \leqq \sum b_{0}\left\{\operatorname{Fr}\left(C_{\lambda}\right)\right\}+b_{0}\left\{\operatorname{Co}\left(A^{\prime}\right) \cup X\right\} .
$$

But $\operatorname{Co}\left(A^{\prime}\right) \cup X=\bar{B}^{\prime}$, and is therefore connected. Accordingly, since $b_{0}(X)>n$, there exist a finite number of components $C_{\lambda}$ of $\operatorname{Co}\left(A^{\prime}\right)$, say $C_{1}, C_{2}, \cdots, C_{k}$, such that $\sum b_{0}\left\{\operatorname{Fr}\left(C_{i}\right)\right\}>n(1 \leqq i \leqq k)$. Let $A=\operatorname{Co}\left(C_{1} \cup C_{2}\right.$ $\cup \ldots \cup C_{k}$ ). It is easy to see that $A$ is connected and open, that the components of $\operatorname{Co}(A)$ are simply the closed sets $C_{i}(1 \leqq i \leqq k)$, and that the components of $\operatorname{Fr}(A)$ are precisely the components of the sets $\operatorname{Fr}\left(C_{i}\right)$. Thus we obtain $b_{0}\{\operatorname{Fr}(A)\}=\sum\left\{b_{0}\left(\operatorname{Fr}\left(C_{i}\right)\right)+1\right\}-1>n+k-1$, where $k-1$ $=b_{0}(\mathrm{Co}(A))$, as desired.

4.4. From the open set $A$ just constructed, we now derive a closed set $A^{*}$ having the same properties. We may assume $b_{0}\left\{\operatorname{Fr}\left(C_{i}\right)\right\}<\infty \quad(1 \leqq i \leqq k)$, since otherwise we merely take $A^{*}=C_{i}$. Thus $\operatorname{Fr}(A)$ will have a finite number of components, say $F_{1}, F_{2}, \cdots, F_{m}$, where $m>k+n$. Take open sets $V_{i} \supset F_{i}$ such that $\bar{V}_{i} \cap \bar{V}_{j}=0$ whenever $i \neq j$, and let $N_{i}$ denote the union of those components of $V_{i} \cap \bar{A}$ which meet $F_{i}$; thus $F_{i} \subset N_{i} \subset V_{i} \cap \bar{A}$, and $N_{i}$ is open relative to $\bar{A}$ (3.2, Corollary). Hence there exist points $x_{i} \in N_{i} \cap A$; and since $A$ is open and connected there exists a closed connected set $L \subset A$ containing all the $m$ points $x_{i}$. Write $E=L \cup \cup\left\{K_{\lambda} \mid K_{\lambda}\right.$ is a component of $A-\bigcup N_{i}$ which meets $L\}$. Clearly $E$ is connected, and it is easy to see that $\bar{E} \subset A$. Finally, define $A^{*}=\bar{E} \cup \cup\left\{P_{\mu} \mid P_{\mu}\right.$ is a component of $A-\bar{E}$ such that $\left.\operatorname{Fr}\left(P_{\mu}\right) \subset \operatorname{Fr}(E)\right\}$. One readily verifies that $A^{*}$ is a closed connected subset of $A$. Further, since in any case $\operatorname{Fr}\left(P_{\mu}\right) \subset \operatorname{Fr}(A) \cup \operatorname{Fr}(E), A-A^{*}$ is a union of components $P_{\nu}$ of $A-\bar{E}$ which are such that $\operatorname{Fr}\left(P_{\nu}\right)$ meets $\operatorname{Fr}(A)$; thus $\mathrm{Co}\left(A^{*}\right)$ is connected relative to $\operatorname{Co}(A)$, and so has at most $k$ components. Again, $x_{i}$ will belong to a component $W_{i}$ (say) of $N_{i} ; W_{i}$ meets $A^{*}$ (in $x_{i}$ ) 
and meets $F_{i} \subset \operatorname{Co}\left(A^{*}\right)$, and so meets $\operatorname{Fr}\left(A^{*}\right)$. Thus $\operatorname{Fr}\left(A^{*}\right)$ meets each $\bar{N}_{i}$; and since $\operatorname{Fr}\left(A^{*}\right) \subset \operatorname{Fr}(E) \subset \bigcup \bar{N}_{i}$, a union of $m$ disjoint closed sets, $\operatorname{Fr}\left(A^{*}\right)$ must have at least $m$ components. Therefore $b_{0}\left\{\operatorname{Fr}\left(A^{*}\right)\right\}$ $>b_{0}\left\{\operatorname{Co}\left(A^{*}\right)\right\}+n$.

\section{Equivalences for spaces without local cut points.}

5.1. Theorem 6. If $S$ has no local cut points, all the properties (i)-(vi) of Theorem 1 (2.2) are equivalent.

It will suffice to prove that (ii)' $\rightarrow$ (i); thus we may assume $r(S)>n$, and must construct a simple open subset $E$ of $S$ such that $b_{0}\{\operatorname{Fr}(E)\}>n$. To avoid obscuring the idea of the proof by "point-set" complications, we shall first carry it out assuming $S$ to be a Peano space, and shall then briefly indicate how the argument would be modified in the general case.

5.2. Proof if $S$ is a Peano space. By Theorem 5a (4.3), there exists an open connected set $A$ such that (i) $\operatorname{Co}(A)$ has only a finite number of components $C_{1}, \cdots, C_{k}$ and (ii) $b_{0}\{\operatorname{Fr}(A)\}>b_{0}\{\operatorname{Co}(A)\}+n$. If $k=1$, there is nothing to prove. If $k>1$, we shall show that $A$ may be replaced by a set $B$ having similar properties but a smaller value of $k$; iteration will then lead to $k=1$, establishing the theorem.

We may assume $b_{0}\left\{\operatorname{Fr}\left(C_{i}\right)\right\}<\infty$, since otherwise we merely take $E=\operatorname{Co}\left(C_{i}\right)$. Hence $\operatorname{Fr}(A)$ consists of a finite number of components $F_{1}, \cdots, F_{m}$, where (as in 4.4) $m>k+n$. Choose connected open sets $U_{i} \supset F_{i}$ such that $\bar{U}_{i} \cap \bar{U}_{j}=0$ whenever $i \neq j$, and choose points $x_{i} \in U_{i} \cap A$. Since $S$ has no local cut points, there exists (see Whyburn [2]) a simple closed curve $J$ in $A$ which contains all the $m$ points $x_{i}$. There exists, for each $i$, a simple $\operatorname{arc} L_{i}$ joining $x_{i}$ to $F_{i}$ in $U_{i}$; and, on replacing $L_{i}$ by a sub-arc if necessary, we can ensure that $L_{i} \cap J$ is a single point, say $y_{i}$, and that $L_{i} \cap F_{i}$ is a single point $z_{i}$. Write $L_{i}^{\prime}=L_{i}-\left(z_{i}\right)$; thus $L_{i}^{\prime} \subset A$. By a suitable change of numbering, we can suppose that $y_{1}, y_{2}, \cdots, y_{m}$ are in cyclic order on $J$, and that $F_{1} \subset C_{1}$ and $F_{2} \subset C_{2}$.

Let $H, K$ denote the two open arcs $\left(y_{1} y_{2}\right)$ of $J$, the points $y_{3}, \cdots, y_{m}$ belonging to $K$. Consider the sets $R=H \cup L_{1} \cup L_{2}, T=K \cup L_{3}^{\prime} \cup \ldots \cup L_{m}^{\prime}$. Clearly $R$ and $T$ are connected sets, $R$ is closed, $R \cap T=0$, and $T \subset A$. Let $B$ be the component of $A-R$ which contains $T$. Since $R \cap A$ is connected, $B$ is simple relative to $A$-that is, $B$ and $A-B$ are both connected; further, $B$ is open; and since the frontier of $B$ relative to $A$ is contained in $R, \operatorname{Fr}(B)$ meets $R$.

We have $\operatorname{Co}(B)=\left\{(A-B) \cup R \cup C_{1} \cup C_{2}\right\} \cup C_{3} \cup \ldots \cup C_{k}$, in which $\left\{(A-B) \cup R \cup C_{1} \cup C_{2}\right\}$ is connected; hence $\operatorname{Co}(B)$ has at most $k-1$ components. And $\operatorname{Fr}(B) \subset \operatorname{Fr}(A) \cup R=\left\{F_{1} \cup R \cup F_{2}\right\} \cup F_{3} \cup \ldots \cup F_{m}$, a union of $m-1$ disjoint closed sets, each of which meets $\operatorname{Fr}(B)$ (for if $i>2, z_{i} \in \bar{T}$ $\left.\cap F_{i} \subset \bar{B} \cap F_{i}=\operatorname{Fr}(B) \cap F_{i}\right)$. Hence $\operatorname{Fr}(B)$ has at least $m-1$ components, so $b_{0}\{\operatorname{Fr}(B)\}>b_{0}\{\operatorname{Co}(B)\}+n$. 
Thus $B$ has all the properties which were required of $A$, and $\operatorname{Co}(B)$ has fewer components than $\mathrm{Co}(A)$. By repeating the argument we finally obtain a simple open set $E$ with these properties; and then $b_{0}\{\operatorname{Fr}(E)\}>n$. Q.E.D.

5.3. Extension to general $S$. In the general case, the arcs used in the above construction will no longer be available; but they can be replaced by suitable closed connected sets, and the argument is then essentially the same as before. The main result to be proved, in order to replace the configuration of arcs used above, is:

(1) Let $A$ be a connected open subset of $S$ (having no local cut points), and let $U_{1}, \cdots, U_{m}$ be open connected sets such that $\bar{U}_{i} \cap \bar{U}_{j}=0(i \neq j)$ and each $U_{i}$ meets $\operatorname{Fr}(A)$. Then there exist connected open sets $V_{i}$ such that (a) $V_{i} \subset A$ and $\bar{V}_{i} \subset U_{i}$, (b) $\operatorname{Fr}\left(V_{i}\right)$ meets $\operatorname{Fr}(A)$, and (c) after a suitable renumbering of the sets $V_{i}$, there exist pairwise disjoint closed connected subsets $H_{1}, \cdots, H_{m}$ of $A$ such that $H_{i}$ meets $\bar{V}_{i}$ and $\bar{V}_{i+1}$, but does not meet $\bar{V}_{j}$ if $j \neq i, i+1\left(i, j=1, \cdots, m ; V_{m+1}=V_{1}\right)$.

This can be proved by induction over $m$, the main lemma being the following sharpening of one due to Ayres and Whyburn (Ayres [1]):

(2) Let $A$ be a connected open set having no cut point, let $E_{1}, E_{2}$ be subsets of $A$ such that $A \cap \bar{E}_{1} \cap \bar{E}_{2}=0$, and let $p, q$ be distinct points of $A$ such that neither of the sets $A \cap \bar{E}_{1}, A \cap \bar{E}_{2}$, disconnects $\left({ }^{15}\right)$ the other from $p$ in $A$. Then there exist two disjoint closed connected sets $H, K$, such that $H$ joins one of $E_{1}$ and $E_{2}$, say $E_{i}$, to $p, K$ joins the other, $E_{j}$, to $q, H \subset A-\bar{E}_{j}$, and $K \subset A-\bar{E}_{i}$.

The proof of (2) follows the lines of the argument in Whyburn [1, pp. 93, 94]. The detailed proofs of (1) and (2) are, however, long and tedious, and are therefore omitted.

\section{Frontier properties and approximation.}

6.1. In subsequent arguments we shall have to replace two open (or closed) sets $A, B$ by approximating closed (or open) ones, and it will be important that this can be done without losing certain frontier properties which $A$ and $B$ may have. As we now show, this can be done; in fact, the relevant frontier properties will actually be improved by the process. Thus, as a byproduct, we shall later be able to state theorems under slightly weaker hypotheses on $A$ and $B$ than would otherwise be the case.

Definition. We shall say that two sets $A, B$ are "strongly approximable by open sets having property $(\mathrm{P})$ " if there exist open sets $U \supset A$ and $V \supset B$ such that every two open sets $A^{*}, B^{*}$ such that $A \subset A^{*} \subset U$ and $B \subset B^{*} \subset V$ have property $(\mathrm{P})\left({ }^{16}\right)$.

(16) A set $X$ "disconnects" sets $Y, Z$ if every connected set which meets both $Y$ and $Z$ must also meet $X$.

(16) Though the theorems which follow all deal with strong approximability, it will be clear that in the applications a much weaker notion of approximability would usually suffice. Cf. 7.8 below. 
6.2. Lemma. If $\operatorname{Fr}(A) \cap \operatorname{Fr}(B) \cap \operatorname{Fr}(A \cup B)=0$, then $A$ and $B$ are strongly approximable by open sets having disjoint frontiers.

There exist open sets $L \supset \operatorname{Fr}(A), M \supset \operatorname{Fr}(B), N \supset \operatorname{Fr}(A \cup B)$ such that $\bar{L} \cap \bar{M} \cap \bar{N}=0$. Write $X=(A \cup L) \cap(A \cup B \cup N), \quad Y=(B \cup M) \cap(A \cup B \cup N)$; it is easy to see that $X$ and $Y$ are open sets containing $A$ and $B$ respectively.

Suppose now that $A^{*}$ and $B^{*}$ are open sets such that $A \subset A^{*} \subset X$ and $B \subset B^{*} \subset Y$; we must prove $\operatorname{Fr}\left(A^{*}\right) \cap \operatorname{Fr}\left(B^{*}\right)=0$. If not, let $x \in \operatorname{Fr}\left(A^{*}\right)$ $\cap \operatorname{Fr}\left(B^{*}\right)$; then $x \in \bar{A}^{*}-A^{*} \subset \bar{X}-A \subset \mathrm{Cl}(A \cup L)-A \subset \bar{L}$. Similarly $x \in \bar{M}$. Again, $x \in \bar{X} \subset \mathrm{Cl}(A \cup B \cup N)$; but $x \notin A$, and similarly $x \notin B$, so that $x \in \bar{N}$. This contradicts the choice of $L, M, N$.

6.3. TheOREM 7. If $A-B$ and $B-A$ are separated $\left.{ }^{17}\right)$ and $\operatorname{Fr}(A) \cap \operatorname{Fr}(B)$ $\cap \operatorname{Fr}(A \cup B)=0$, then, given any open set $W \supset A \cap B, A$ and $B$ are strongly approximable by open sets whose frontiers are disjoint and whose intersection is contained in $W$.

With the same sets $L, M, N$ as in the lemma preceding, write $U^{*}=A$ $\cup\{N \cap(L-\bar{B})\} \cup W, V^{*}=B \cup\{N \cap(M-\bar{A})\} \cup W$. It is not hard to see that $U^{*}$ and $V^{*}$ are open; and clearly $A \subset U^{*}, B \subset V^{*}$, and $U^{*} \cap V^{*}=W$. The sets $U=X \cap U^{*}$ and $V=Y \cap V^{*}$ are now the open neighbourhoods of $A$ and $B$ required; in fact, if $A^{*}, B^{*}$ are open and $A \subset A^{*} \subset U^{*}$ and $B \subset B^{*} \subset V$, we have $\operatorname{Fr}\left(A^{*}\right) \cap \operatorname{Fr}\left(B^{*}\right)=0$, from the lemma, and $A^{*} \cap B^{*} \subset U^{*} \cap V^{*}=W$.

6.4. Remark. The conditions on $A$ and $B$ in Theorem 7-that $A-B$ and $B-A$ are separated and $\operatorname{Fr}(A) \cap \operatorname{Fr}(B) \cap \operatorname{Fr}(A \cup B)=0$-are together equivalent to the single condition $\operatorname{Fr}(A) \cap \operatorname{Fr}(B) \subset A \cap B \cap \operatorname{Int}(A \cup B)$. They therefore certainly hold if $\operatorname{Fr}(A) \cap \operatorname{Fr}(B)=0$. Conversely, they imply $\operatorname{Fr}(A) \cap \operatorname{Fr}(B)=0$ if either $A$ or $B$ is open.

6.5. Theorem 7a. If $A-B$ and $B-A$ are separated, and also $A \cap B$ and $\operatorname{Co}(A \cup B)$ are separated, then, given any open set $W$ containing $A \cap B, A$ and $B$ are strongly approximable by open sets $A^{*}, B^{*}$, satisfying $A^{*} \cap B^{*} \subset W$ and $\operatorname{Fr}\left(A^{*}\right) \cap \operatorname{Fr}\left(B^{*}\right) \cap \operatorname{Fr}\left(A^{*} \cap B^{*}\right)=0$.

There exist open sets $L, M, X, Y$ such that $A-B \subset L, B-A \subset M$, $L \cap M=0, A \cap B \subset X, \operatorname{Co}(A \cup B) \subset Y$, and $X \cap Y=0$. (Here, for the first time, we use the complete normality of $S$.) Write $U=L \cup(X \cap W), V=M \cup(X \cap W)$; clearly $U, V$ are open sets containing $A, B$, respectively, and $U \cap V \subset W \cap X$. Let $A^{*}, B^{*}$ be any open sets such that $A \subset A^{*} \subset U$ and $B \subset B^{*} \subset V$. Clearly $A^{*} \cap B^{*} \subset W$; and we have $\operatorname{Fr}_{2}\left(A^{*}\right) \cap \operatorname{Fr}\left(B^{*}\right) \cap \operatorname{Fr}\left(A^{*} \cap B^{*}\right) \subset \operatorname{Co}\left(A^{*}\right) \cap \operatorname{Co}\left(B^{*}\right)$ $\cap \mathrm{Cl}\left(A^{*} \cap B^{*}\right) \subset Y \cap \bar{X}=0$.

6.6. REMARKS.

(1) The hypotheses on $A$ and $B$ in Theorem 7 a are implied by those in

(17) This condition (introduced by Wallace [1]) will hold if $A$ and $B$ are both closed, or both open, relative to $A \cup B$, or if $\operatorname{Fr}(A) \cap \operatorname{Fr}(B)=0$, and so on. 
Theorem 7-an implication which becomes an equivalence if $A$ and $B$ are both closed. Again, if they are satisfied by $A$ and $B$, they are satisfied also by $\operatorname{Co}(A)$ and $\operatorname{Co}(B)$; hence they are also implied by: $\{A-B$ and $B-A$ are separated and $\operatorname{Fr}(A) \cap \operatorname{Fr}(B) \cap \operatorname{Fr}(A \cap B)=0\}$-an implication which becomes an equivalence if $A$ and $B$ are both open.

(2) The converse of Theorem 7 a is also true, provided that $S$ is metrisable (or, more generally, has a locally countable base-some such proviso is essential) and has no isolated points. The converse of Theorem 7 is false in general, but holds if $A$ and $B$ are closed.

(3) The arguments in the present section made no use of the connectedness or local connectedness of $S$; they are therefore valid (except for the converse of Theorem $7 \mathrm{a}$ ) in an arbitrary completely normal space. Further, the only use made of complete normality-and the only essential use of it in the whole paper-was in proving Theorem $7 \mathrm{a}$; elsewhere, normality would have sufficed. Thus our assumptions on $S$ could be weakened throughout to requiring only that $S$ be a non-empty, connected, locally connected, and normal $\left(T_{1}\right)$ space, provided that two minor adjustments are made in what follows: (a) every appeal to Theorem 7 a must be replaced by an appeal to Theorem 7, the hypotheses on $A$ and $B$ being strengthened accordingly, and (b) for general sets (neither open nor closed), $b_{0}(X)$ should be replaced by a similar function based on the "normal components" of $X$, which are defined in an obvious way in terms of the notion of "normal connectedness" given in $[\mathrm{U}, 4.3]$.

\section{Connected sets with "almost disjoint" frontiers.}

7.1. In $[\mathrm{U}]$ it was shown that the defining property of unicoherence could be extended to two connected sets which were open and covered $S$, or more generally which had disjoint frontiers (and were not necessarily open). Here we shall obtain a similar extension; but the process can now (in view of $\S 6$ ) be carried even further, applying to sets which can be approximated by sets having disjoint frontiers (Theorem 9, 7.5) - a fairly inclusive result, since it includes also, for example, the case in which the two connected sets are closed and cover $S$. It readily follows that the sets used to define $r(S)$ could be taken to be open, instead of closed, without affecting the definition (Theorem 10, 7.7). We conclude $\$ 7$ by showing that Theorem 9 also holds under modified conditions, and deducing some implications between frontier relations for the case in which $S$ is unicoherent.

7.2. The starting point is a generalisation of the "extension theorem" [U, Theorem 4, 4.8]:

TheOREM 8. Let $A$ and $B$ be any sets, not both empty, such that $\operatorname{Fr}(A) \cap \operatorname{Fr}(B) \cap \operatorname{Fr}(A \cup B)=0$. Then there exist sets $A^{*}, B^{*}$, such that $A^{*} \supset A, B^{*} \supset B, A^{*} \cup B^{*}=S, A \cap B$ is both open and closed relative to $A^{*} \cap B^{*}$, $A^{*}$ and $B^{*}$ are connected relative to $A$ and $B$ respectively, and $\operatorname{Fr}\left(A^{*}\right) \cap \operatorname{Fr}\left(B^{*}\right)$ $\subset \operatorname{Fr}(A) \cap \operatorname{Fr}(B)$. Moreover, $A^{*}$ and $B^{*}$ can be chosen to be closed if $A$ and $B$ 
are closed, and open if $A$ and $B$ are open $\left({ }^{18}\right)$.

Proof. Write $C=\operatorname{Co}(A \cup B), F_{1}=\operatorname{Fr}(C) \cap \operatorname{Fr}(A)$, and $F_{2}=\operatorname{Fr}(C) \cap \operatorname{Fr}(B)$; thus $F_{1} \cap F_{2}=0$ and $F_{1} \cup F_{2}=\operatorname{Fr}(C)$. The case in which $F_{1} \cup F_{2}=0$ is easily seen to be trivial; hence we may apply Theorem 2 (3.4), obtaining closed sets $H_{1}, H_{2}$, such that $H_{i} \supset F_{i}$ and $H_{i}$ is connected relative to $F_{i}(i=1,2)$, $H_{1} \cup H_{2}=\bar{C}, H_{1} \cap H_{2} \subset \operatorname{Int}(C)$, and $\operatorname{Fr}\left(H_{1}\right) \cap \operatorname{Fr}\left(H_{2}\right)=0$. Now define $A^{*}=A$ $\cup H_{1}, B^{*}=B \cup H_{2}$. Clearly $A^{*}, B^{*}$ are connected relative to $A, B$, respectively, and $A^{*} \cup B^{*}=S$. Further, it is easy to see that $A^{*} \cap B^{*}=(A \cap B) \cup\left(H_{1} \cap H_{2}\right)$, where $H_{1} \cap H_{2} \subset \operatorname{Ext}(A \cup B)$, so that $A \cap B$ is both open and closed relative to $A^{*} \cap B^{*}$. And we have $\operatorname{Fr}\left(A^{*}\right) \cap \operatorname{Fr}\left(B^{*}\right) \subset\left\{\operatorname{Fr}(A) \cup \operatorname{Fr}\left(H_{1}\right)\right\} \cap\{\operatorname{Fr}(B)$ $\left.\cup \operatorname{Fr}\left(H_{2}\right)\right\} \subset \operatorname{Fr}(A) \cap \operatorname{Fr}(B)$ since $\operatorname{Fr}\left(H_{1}\right) \cap \operatorname{Fr}\left(H_{2}\right)=0$ and $H_{1} \cap F_{2}=0=H_{2} \cap F_{1}$.

Obviously $A^{*}$ will be closed if $A$ is, and similarly for $B^{*}$. All that remains is to deal with the case in which $A$ and $B$ are both open. Here the definition of $A^{*}$ and $B^{*}$ must be modified. There exists an open set $U$, connected relative to $H_{1} \cap H_{2}$, such that $H_{1} \cap H_{2} \subset U$ and $\bar{U} \subset \operatorname{Int}(C)$. Replace $A^{*}$ by $A^{*} \cup U$ and $B^{*}$ by $B^{*} \cup U$; it is easy to verify that these sets are open and have all the properties required.

REMARK. It is also easy to see that the (unmodified) sets will satisfy: $A^{*}-B^{*}$ and $B^{*}-A^{*}$ are separated if $A-B$ and $B-A$ are separated.

7.3. Lemma. If $A$ and $B$ are closed connected sets such that $\operatorname{Fr}(A) \cap \operatorname{Fr}(B)$ $\cap \operatorname{Fr}(A \cup B)=0$, then $b_{0}(A \cap B) \leqq r(S)$.

For we have only to apply Theorem 8 , giving $b_{0}(A \cap B) \leqq b_{0}\left(A^{*} \cap B^{*}\right)$ $\leqq r(S)$.

7.4. Lemma. If $A$ and $B$ are open connected sets such that $\operatorname{Fr}(A) \cap \operatorname{Fr}(B)$ $\cap \operatorname{Fr}(A \cap B)=0$, then $b_{0}(A \cap B) \leqq r(S)$.

We deduce this from 7.3 by "approximation." Suppose the lemma is false; then $r(S)$ is finite, and equal to $n$, say, and we can pick points $p_{1}, p_{2}, \cdots, p_{n+2}$ belonging to different components of $A \cap B$. There exist closed connected sets $L \subset A$ and $M \subset B$ both of which contain all the $n+2$ points $p_{i}$. Now, the closed sets $\operatorname{Co}(A)$ and $\operatorname{Co}(B)$ satisfy the hypotheses of Theorem 7 (6.3), and $\operatorname{Co}(L)$ and $\operatorname{Co}(M)$ are open sets containing them. Hence (Theorem 7) there exist open sets $X, Y$, with disjoint frontiers, such that $\operatorname{Co}(A) \subset X \subset \mathrm{Co}(L)$ and $\operatorname{Co}(B) \subset Y \subset \mathrm{Co}(M)$. Let $H$ be the component of $\mathrm{Co}(X)$ which contains $L$, and similarly let $K$ be the component of $\operatorname{Co}(Y)$ which contains $M$. Then $H$ and $K$ are closed, and $\operatorname{Fr}(H) \cap \operatorname{Fr}(K) \subset \operatorname{Fr}(X)$ $\cap \operatorname{Fr}(Y)=0$. By $7.3, b_{0}(H \cap K) \leqq n$. But $L \cap M \subset H \cap K \subset A \cap B$; hence some

$\left.{ }^{18}\right)$ By analogy with $[\mathrm{U}, 4.8]$, one might expect that the further conditions $A^{*} \cap B^{*}$ $=A \cap B, \operatorname{Fr}\left(A^{*}\right) \subset \operatorname{Fr}(A)$ and $\operatorname{Fr}\left(B^{*}\right) \subset \operatorname{Fr}(B)$ could also be imposed; but it is easy to see from examples that this is impossible, in general. 
two of the points $p_{i}$ must belong to the same component of $H \cap K$, and therefore to the same component of $A \cap B$ - a contradiction.

7.5. Theorem 9. Let $A$ and $B$ be any connected sets such that $A-B$ and $B-A$ are separated, and also $A \cap B$ and $\operatorname{Co}(A \cup B)$ are separated. Then $b_{0}(A \cap B) \leqq r(S)$.

This follows from 7.4 by "approximation" again. Let $n$ be any nonnegative integer such that $n \leqq b_{0}(A \cap B)$; it will suffice to prove that $n \leqq r(S)$. We can write $A \cap B=C_{1} \cup C_{2} \cup \ldots \cup C_{n+1}$, where the sets $C_{i}$ are non-empty and every two of them are separated. Hence there exist open sets $W_{i} \supset C_{i}$ such that $W_{i} \cap W_{j}=0$ whenever $i \neq j$. Let $W=U W_{i}$, and apply Theorem 7a (6.5); there will exist open sets $A^{*} \supset A$ and $B^{*} \supset B$ such that $A^{*} \cap B^{*} \subset W$ and $\operatorname{Fr}\left(A^{*}\right) \cap \operatorname{Fr}\left(B^{*}\right) \cap \operatorname{Fr}\left(A^{*} \cap B^{*}\right)=0$. Let $U, V$ be the components of $A^{*}, B^{*}$ which contain $A, B$ respectively; then, since $U C_{i} \subset U \cap V \subset \cup W_{i}$, we have $b_{0}(U \cap V) \geqq n$. But it is easy to see that $\operatorname{Fr}(U) \cap \operatorname{Fr}(V) \cap \operatorname{Fr}(U \cap V)=0$; hence $b_{0}(U \cap V) \leqq r(S)$, from 7.4 , and the result follows.

7.6. Remark. The hypotheses on $A$ and $B$ in Theorem 9 are implied by the hypotheses in 7.3 or 7.4 , and also hold whenever either $\operatorname{Fr}(A) \cap \operatorname{Fr}(B)=0$, or $A-B$ and $B-A$ are separated (for example, $A$ and $B$ both open or closed) and $\operatorname{Fr}(A \cap B) \cap \operatorname{Fr}(A \cup B)=0$ (for example, $A \cup B=S$ ).

7.7. Let us now modify the definition of $r(S)$ by writing

$r_{0}(S)=\sup \left\{b_{0}(A \cap B) \mid A, B\right.$ are open, connected, and $\left.A \cup B=S\right\}$,

$r_{d}(S)=\sup \left\{b_{0}(A \cap B) \mid A-B\right.$ and $B-A$ are separated, $A, B$ are connected,

THEOREM 10. $r_{0}(S)=r_{d}(S)=r(S)$. and $A \cup B=S\}$.

In fact, it is trivial that $r_{0}(S) \leqq r_{d}(S)$, it is easy to see that $r(S) \leqq r_{0}(S)$ (this is shown in effect by the argument at the beginning of 4.3 ), and it follows from Theorem 9 (7.5) that $r_{d}(S) \leqq r(S)$.

7.8. Modifications. As one would expect, the hypotheses under which Theorem 9 was proved can be modified in various ways; for example, it would suffice that the sets $A, B$ be sufficiently well approximable by connected sets satisfying those hypotheses. We shall here carry out one such (but less trivial) modification which will be needed later, and which has the feature that in the "interesting" case the modification is only apparent.

We need as a lemma the following modification of Theorem 8 (7.2):

LEMMA. Let $U$ and $V$ be arbitrary subsets of $S$, and let $X$ be a subset of $U \cap V$ such that (a) $X$ is closed relative to $U \cap V$, (b) $X$ is open, and (c) $\operatorname{Fr}(U)$ $\cap \operatorname{Fr}(V) \cap \operatorname{Fr}(X)=0$. Then there exist sets $U^{*}, V^{*}$ such that $U^{*} \supset U, V^{*} \supset V$, $U^{*}$ and $V^{*}$ are connected relative to $U$ and $V$ respectively, $X$ is closed relative to $U^{*} \cap V^{*}$, and $\operatorname{Fr}\left(U^{*}\right) \cap \operatorname{Fr}\left(V^{*}\right) \cap \operatorname{Fr}\left(U^{*} \cap V^{*}\right)=0$. Moreover, $U^{*}$ and $V^{*}$ can be chosen to be open if $U$ and $V$ are open. 
Proof. Write $Y=(U \cap V)-X$; thus $\bar{X} \cap Y=0$. Let $Y^{*}$ be the union of all components of $\operatorname{Co}(\bar{X})$ which meet $Y ; Y^{*}$ is open, connected relative to $Y$, contains $Y$, and is disjoint from $X$. We have only to define $U^{*}=U \cup Y^{*}$, $V^{*}=V \cup Y^{*}$; the verification of the stated properties is immediate. Incidentally, it is also clear that $U^{*}-V^{*}$ and $V^{*}-U^{*}$ will be separated if $U-V$ and $V-U$ are, and that $U^{*}$ and $V^{*}$ will be closed if $U$ and $V$ are, though in this last case the whole situation is trivial (because $X=0$ or $S$ ).

COROLLARY. If $U$ and $V$ are connected open sets, and $X$ is a relatively openclosed subset of $U \cap V$ such that $\operatorname{Fr}(U) \cap \operatorname{Fr}(V) \cap \operatorname{Fr}(X)=0$, then $b_{0}(X) \leqq r(S)$; and if further $b_{0}(X)=r(S)<\infty$, then $X=U \cap V$.

For, on applying 7.4 to the sets $U^{*}, V^{*}$ defined above, we obtain $b_{0}\left(X \cup Y^{*}\right) \leqq r(S)$. Since $X$ and $Y^{*}$ are disjoint open sets, this gives $b_{0}(X)$ $\leqq r(S)$, with finite equality only if $Y^{*}=0$ and therefore $Y=0$.

7.9. THEOREM 11. If $A$ and $B$ are connected sets satisfying $\operatorname{Fr}(A) \cap \operatorname{Fr}(B)$ $\cap \operatorname{Fr}(A \cap B)=0$, then $b_{0}(A \cap B) \leqq r(S)$; and if further $b_{0}(A \cap B)=r(S)<\infty$, then $\operatorname{Fr}(A) \cap \operatorname{Fr}(B)=0$.

Suppose $b_{0}(A \cap B) \geqq n \geqq 0$; to prove the first part of the theorem it will evidently suffice to deduce that $n \leqq r(S)$. We can write $A \cap B=\bigcup E_{i}$ $(1 \leqq i \leqq n+1)$, a union of non-empty sets every two of which are separated, and can take pairwise disjoint open sets $W_{i} \supset E_{i}$. Let $U W_{i}=W$.

There exist open sets $L, M, N$ such that $L \supset \operatorname{Fr}(A), M \supset \operatorname{Fr}(B)$, $N \supset \operatorname{Fr}(A \cap B)$, and $\bar{L} \cap \bar{M} \cap \bar{N}=0$. Again, we have $\mathrm{Cl}(A-B) \cap \mathrm{Cl}(B-A)$ $\cap \mathrm{Cl}(A \cap B) \subset \operatorname{Fr}(A) \cap \operatorname{Fr}(B) \cap \operatorname{Fr}(A \cap B)=0$, so that there exist open sets $P, Q, R$ such that $P \supset \mathrm{Cl}(A-B), Q \supset \mathrm{Cl}(B-A), R \supset A \cap B$, and, $P \cap Q \cap R=0$. Write $X=\{(A \cap B) \cup N\} \cap R \cap W, A^{*}=(A \cup L) \cap(P \cup X)$, and $B^{*}=(B \cup M) \cap(Q \cup X)$. It is easy to see that $X$ is an open set containing $A \cap B$, from which it follows readily that $A^{*}$ and $B^{*}$ are open sets containing $A$ and $B$ respectively. Let $C, D$ be the components of $A^{*}, B^{*}$ which contain the respective sets $A, B$. Thus we have $A \cap B \subset C \cap D \subset A^{*} \cap B^{*} \subset(P \cup X)$ $\cap(Q \cup X)=(P \cap Q) \cup X$; so, on writing $C \cap D \cap P \cap Q=H$ and $C \cap D \cap X=K$, we have $C \cap D=H \cup K$, a union of two disjoint open sets (for $H \subset P \cap Q$ and $K \subset X \subset R)$, of which $K$ contains $A \cap B$.

Now it can be verified that $\operatorname{Fr}(C) \cap \operatorname{Fr}(D) \cap \operatorname{Fr}(K) \subset \mathrm{Cl}(\mathrm{Co}(A))$ $\cap \mathrm{Cl}(\mathrm{Co}(B)) \cap \bar{L} \cap \bar{M} \cap\{\mathrm{Cl}(A \cap B) \cup \bar{N}\}=0$. Hence the corollary to the preceding lemma shows that $b_{0}(K) \leqq r(S)$, and if $b_{0}(K)=r(S)<\infty$, then $H=0$.

Since $A \cap B \subset K \subset X$, we have $U E_{i} \subset K \subset \cup W_{i}$, whence $b_{0}(K) \geqq n$. Thus $n \leqq r(S)$, proving the first part of the theorem.

But if $b_{0}(A \cap B)=r(S)<\infty$, we may suppose that $n=b_{0}(A \cap B)$ in the preceding, and so have $H=0$. But it can be verified that $\operatorname{Fr}(A) \cap \operatorname{Fr}(B) \subset C$ $\cap D \cap P \cap Q=H$; thus $\operatorname{Fr}(A) \cap \operatorname{Fr}(B)=0$, completing the proof.

7.10. Similarly one can prove: 
TheOREM 11a. If $A$ and $B$ are connected sets such that $A-B$ and $B-A$ are separated, and $\operatorname{Fr}(A \cap B) \cap \operatorname{Fr}(A \cup B)=0$, then $b_{0}(A \cap B) \leqq r(S)$; and if further $b_{0}(A \cap B)=r(S)<\infty$, then $\operatorname{Fr}(A) \cap \operatorname{Fr}(B) \cap \operatorname{Fr}(A \cup B)=0$.

The first part of this statement is, of course, contained in Theorem 9 (7.5); however, Theorem 11a can be established (with a suitable modification of the definition of $b_{0}$; cf. 6.6(3)) assuming only the normality, and not the complete normality, of $S$, whereas Theorem 9 cannot be carried over in this way as its proof uses Theorem $7 \mathrm{a}(6.5)$.

7.11. As an immediate consequence of Theorem 11 we have

(1) If $S$ is unicoherent, and $A$ and $B$ are intersecting connected sets, then $\{\operatorname{Fr}(A) \cap \operatorname{Fr}(B) \cap \operatorname{Fr}(A \cap B)=0\} \rightarrow\{\operatorname{Fr}(A) \cap \operatorname{Fr}(B)=0\}$.

Similarly, Theorem 11a gives.

(2) If $S$ is unicoherent, and $A$ and $B$ are intersecting connected sets such that $A-B$ and $B-A$ are separated, then $\{\operatorname{Fr}(A \cap B) \cap \operatorname{Fr}(A \cup B)=0\}$ $\rightarrow\{\operatorname{Fr}(A) \cap \operatorname{Fr}(B) \cap \operatorname{Fr}(A \cup B)=0\}$.

It readily follows that if $S$ is unicoherent and $A$ and $B$ are open intersecting connected sets, the four properties $\operatorname{Fr}(A \cap B) \cap \operatorname{Fr}(A \cup B)=0$, $\operatorname{Fr}(A) \cap \operatorname{Fr}(B) \cap \operatorname{Fr}(A \cup B)=0, \operatorname{Fr}(A) \cap \operatorname{Fr}(B) \cap \operatorname{Fr}(A \cap B)=0$, and $\operatorname{Fr}(A)$ $\cap \operatorname{Fr}(B)=0$ are all equivalent.

Conversely, these implications are characteristic of unicoherence. In fact, if $S$ is not unicoherent, it is easy to construct two intersecting connected open sets $A, B$ such that $\operatorname{Fr}(A \cap B) \cap \operatorname{Fr}(A \cup B)=\operatorname{Fr}(A) \cap \operatorname{Fr}(B) \cap \operatorname{Fr}(A \cap B)=0$, and $\operatorname{Fr}(A) \cap \operatorname{Fr}(B) \cap \operatorname{Fr}(A \cup B)=\operatorname{Fr}(A) \cap \operatorname{Fr}(B) \neq 0$.

\section{Intersections of two connected sets and their frontiers.}

8.1. We conclude by generalising Theorem 5 of $[\mathrm{U}, 5.1]$, concerning two "nearly" connected sets whose frontiers do intersect. The result is the "expected" one:

THEOREM 12. If $\bar{A}$ and $\bar{B}$ are connected, and $\operatorname{Fr}(A) \cap \operatorname{Fr}(B) \neq 0$, then $b_{0}(\bar{A} \cap \bar{B}) \leqq b_{0}\{\operatorname{Fr}(A) \cap \operatorname{Fr}(B)\}+r(S)$.

Proof. Suppose $b_{0}(A \cap B) \geqq n \geqq 0$; it will suffice to deduce that $n \leqq b_{0}\{\operatorname{Fr}(A) \cap \operatorname{Fr}(B)\}+r(S)$. We can write $A \cap B=C_{1} \cup C_{2} \cup \ldots \cup C_{n+1}$, where the sets $C_{i}$ are closed, non-empty, and pairwise disjoint; and we can suppose the notation to be such that $C_{1}, \cdots, C_{m}$ meet $\operatorname{Fr}(A) \cap \operatorname{Fr}(B)$ and $C_{m+1}, \cdots, C_{n+1}$ do not, for some $m$ such that $1 \leqq m \leqq n+1$. There exist open sets $W_{i} \supset C_{i}(1 \leqq i \leqq n+1), H \supset \operatorname{Fr}(A), K \supset \operatorname{Fr}(B)$, such that the sets $\bar{W}_{i}$ are pairwise disjoint and further $\bar{W}_{j} \cap \bar{H} \cap \bar{K}=0$ if $j>m$. Write $W=\cup W_{j}$ $(m<j \leqq n+1)$, and let $H_{1}$ denote the union of those components of $H \cap W$ which meet $\bar{A}$. Similarly, let $K_{1}$ denote the union of the components of $K \cap W$ which meet $\bar{B}$. Write $U=\bar{A} \cup H_{1}, V=\bar{B} \cup K_{1}$, and $X=U \cap V \cap W$. Clearly $U$ and $V$ are connected; and it is easy to see that $U \cap W$ and $V \cap W$ are open, so that $X$ is open. Again, $X$ is closed relative to $U \cap V$, since $(U \cap V)-X=(\bar{A} \cap \bar{B})-W=C_{1} \cup \ldots \cup C_{m}=(U \cap V) \cap\left(W_{1} \cup \ldots \cup W_{m}\right)$, 
which is open relative to $U \cap V$. Lastly, $\operatorname{Fr}(U) \cap \operatorname{Fr}(V) \cap \operatorname{Fr}(X) \subset \bar{H} \cap \bar{K} \cap \bar{W}$ $=0$. Hence, by the lemma in 7.8 , there exist connected sets $U^{*} \supset U$ and $V^{*} \supset V$, such that $X$ is both open and closed relative to $U^{*} \cap V^{*}$, and $\operatorname{Fr}\left(U^{*}\right) \cap \operatorname{Fr}\left(V^{*}\right) \cap \operatorname{Fr}\left(U^{*} \cap V^{*}\right)=0$.

By Theorem $11(7.9), b_{0}\left(U^{*} \cap V^{*}\right) \leqq r(S)$. Since the sets $X$ and $\left(U^{*} \cap V^{*}\right)$ $-X$ are separated, and the latter is non-empty (containing $C_{1}$ at least), it follows that $X$ has at most $r(S)$ components. But, by construction, we have $C_{m+1} \cup \ldots \cup C_{n+1} \subset X \subset W_{m+1} \cup \ldots \cup W_{n+1}$, so that $X$ has at least $n-m+1$ components. Thus $n-m+1 \leqq r(S)$.

Again, for each $i \leqq m$, there exists a component $F_{i}$ of $\operatorname{Fr}(A) \cap \operatorname{Fr}(B)$ which meets $C_{i}$; clearly $F_{i} \subset C_{i}$, and so $\operatorname{Fr}(A) \cap \operatorname{Fr}(B)$ has at least $m$ components. The theorem now follows, since we have $n \leqq b_{0}\{\operatorname{Fr}(A) \cap \operatorname{Fr}(B)\}+r(S)$.

8.2. REMARKs. (1) We have shown incidentally that, under the hypotheses of Theorem 12, if $b_{0}(\bar{A} \cap \bar{B})<\infty$, then all but at most $r(S)$ of the components of $\bar{A} \cap \bar{B}$ meet $\operatorname{Fr}(A) \cap \operatorname{Fr}(B)$. (Cf. [U, 5.2, Corollary 1].)

(2) Theorem 12 gives an alternative characterisation of $r(S)$. In fact, if $r(S)>n \geqq 0$, there exist open connected sets $A, B$ such that $\operatorname{Fr}(A) \cap \operatorname{Fr}(B)$ consists of a single point and $b_{0}(\bar{A} \cap \bar{B})>n$. (Cf. [U, 5.6].)

\section{BIBLIOGRAPHY}

\section{W. L. Ayres}

1. Concerning continuous curves in metric space, Amer. J. Math. vol. 51 (1929) pp. 577-594.

\section{S. EILENBERG}

1. Sur les espaces multicoherents I, Fund. Math. vol. 27 (1936) pp. 153-190.

2. Sur les espaces multicoherents II, Fund. Math. vol. 29 (1937) pp. 101-122.

3. Sur la multicohérence des surfaces closes, Comptes Rendus de l'Académie des Sciences de Warsaw vol. 30 (1937) pp. 109-111.

D. W. Hall and A. D. Wallace

1. Some invariants under monotone transformations, Bull. Amer. Math. Soc. vol. 45 (1939) pp. 294-295.

C. KURATOWSKI

1. Topologie I, Warsaw, 1933.

2. Sur les continus de Jordan et le théorème de M. Brouwer, Fund. Math. vol. 8 (1926) pp. $137-150$.

A. H. Stone

1. Incidence relations in unicoherent spaces, Trans. Amer. Math. Soc. vol. 65 (1949) pp. 427-447.

A. D. Wallace

1. Separation spaces, Ann. of Math. vol. 42 (1941) pp. 687-697.

G. T. WHYBURN

1. Analytic topology, Amer. Math. Soc. Colloquium Publications, vol. 28, 1942.

2. Concerning the proposition that every closed compact totally disconnected set of points is a subset of an arc, Fund. Math. vol. 18 (1932) pp. 47-60.

W. A. Wilson

1. A property of continua equivalent to local connectivity, Bull. Amer. Math. Soc. vol. 36 (1930) pp. 85-88.

Trinity College, Cambridge University, Cambridge, England. 